\title{
VARIABILIDAD INTRAANUAL DE LA OSCILACIÓN DEL MEDITERRÁNEO OCCIDENTAL (WEMO) Y OCURRENCIA DE EPISODIOS TORRENCIALES EN CATALUÑA
}

\author{
Joan Albert LÓPEZ-BUSTINS ${ }^{1}$, Javier MARTÍN-VIDE ${ }^{1}$, Marc PROHOM² \\ y María José CORDOBILLA ${ }^{1}$ \\ ${ }^{1}$ Grup de Climatologia, Facultat de Geografia i Història, Universitat de Barcelona. \\ ${ }^{2}$ Departament de Climatologia, Servei Meteorologic de Catalunya. \\ jlopezbustins@ub.edu, jmartinvide@ub.edu,.mprohom@meteo.cat, mj.cordobilla@ub.edu
}

\section{RESUMEN}

Varios estudios anteriores han demostrado que el índice de la Oscilación del Mediterráneo Occidental (WeMOi) tiene una correlación estadísticamente significativa con los totales pluviométricos de la vertiente oriental de la Península Ibérica. En este trabajo se presenta un calendario del WeMOi a partir de los valores medios obtenidos por períodos de diez días, y se relaciona con la ocurrencia de episodios torrenciales en las provincias costeras de Cataluña (Girona, Barcelona y Tarragona). A lo largo de los 64 años del período de estudio 1950-2013, se han detectado 375 días (5,9 por año) con precipitación torrencial ( $\geq 100 \mathrm{~mm}$ en $24 \mathrm{~h}$ ) y 36 episodios de torrencialidad extrema ( $\geq 200 \mathrm{~mm}$ en $24 \mathrm{~h}$ ) (0,6 por año). Se ha obtenido una buena correspondencia entre los valores intraanuales del WeMOi y la frecuencia de episodios. Los valores más negativos del WeMOi tienen lugar en otoño, concretamente, durante la segunda década de octubre (del 11 al 20 de octubre). En esa década se registra la máxima frecuencia de episodios de 100 y $200 \mathrm{~mm}$ en 24 h en el área de estudio. En el análisis también se consideran dos subperíodos de estudio de 32 años (1950-1981 y 1982-2013) para detectar variaciones tanto en el calendario del WeMOi como en la frecuencia de episodios torrenciales. El segundo subperíodo muestra en la mayoría de décadas valores del WeMOi más negativos que el primer subperíodo, especialmente, a finales de otoño y principios de invierno. En correspondencia con ello se observa un aumento de la frecuencia de episodios torrenciales en el segundo subperíodo (222 días) respecto al primero (153 días). WeMO.

Palabras clave: Cataluña, Precipitación Torrencial, Variabilidad Intraanual,

\begin{abstract}
Several previous studies have demonstrated that the Western Mediterranean Oscillation index (WeMOi) has a statistically significant correlation with total rainfall amount over eastern Iberia. In the present paper, we present a WeMOi calendar using mean daily values derived from 10-day periods, relating it to the occurrence of heavy rainfall episodes over the coastal provinces of Catalonia (Girona, Barcelona and Tarragona). During the 1950-2013 study period, i.e. 64 years, 375 days (5.9 per year) were found to present heavy rainfall ( $\geq 100 \mathrm{~mm}$ in $24 \mathrm{~h}$ ) and 36 days to present extremely heavy rainfall ( $\geq 200 \mathrm{~mm}$ in $24 \mathrm{~h}$ ) ( 0.6 per year). We found a good match
\end{abstract}


between the intra-annual WeMOi values and the occurrence of heavy rainfall. The most negative WeMOi values are observed in autumn, especially during the second decade of October (from $11^{\text {th }}$ to $20^{\text {th }}$ ). In this decade, the rainfall episodes of 100 and $200 \mathrm{~mm}$ in $24 \mathrm{~h}$ over the study area accumulate the highest frequency. The study analysis also considers two subperiods of 32 years (1950-1981 and 1982-2013) in order to detect variations both in the WeMOi calendar and in the frequency of heavy rainfall episodes. In most decades, the second subperiod exhibits lower WeMOi values than the first subperiod, particularly at the end of autumn and the start of winter. Accordingly, we observed an increase in the frequency of heavy rainfall episodes during the second subperiod (222 days) compared with the first subperiod (153 days).

Key words: Catalonia, Heavy Rainfall, Intra-annual Variability, WeMO.

\section{INTRODUCCIÓN}

El clima mediterráneo se distingue del resto de climas mundiales por la ocurrencia de un período de lluvias escasas o nulas durante la estación cálida (Kottek et al., 2006). El conjunto de la cuenca mediterránea presenta un máximo pluviométrico en invierno y un mínimo en verano. No obstante, en algunos sectores de la cuenca el régimen de precipitaciones es algo distinto. Es el caso de la fachada oriental de la Península Ibérica, donde los meses más lluviosos son los próximos a los equinoccios (De Luis et al., 2010). Incluso, en el litoral y prelitoral mediterráneo de la Península el otoño es apreciablemente más lluvioso que la primavera (Martín-Vide y OlcinaCantos, 2001). Los episodios de lluvias intensas y torrenciales suelen acontecer en estas franjas litoral y prelitoral durante los meses otoñales, lo que incrementa sus totales mensuales.

Martín-Vide y López-Bustins (2006) definieron un índice de teleconexión centrado en la cuenca occidental del Mediterráneo, cuyo primer ensayo se presentó en el III Congreso de la Asociación Española de Climatología (AEC) (Martín-Vide, 2002), que explica el comportamiento pluviométrico de la fachada mediterránea de la Península Ibérica: el índice de la Oscilación del Mediterráneo Occidental (Western Mediterranean Oscillation index, WeMOi). Esta región oriental de la Península se halla a sotavento de los flujos del Atlántico y presenta una débil influencia de la Oscilación del Atlántico Norte (North Atlantic Oscillation, NAO), el patrón de variabilidad de baja frecuencia que rige el tiempo meteorológico en invierno sobre la mayor parte de la Europa Occidental (Hurrell, 1995).

En el presente estudio se relaciona la variabilidad intraanual del WeMOi con la ocurrencia de episodios torrenciales $(\geq 100 \mathrm{~mm}$ en $24 \mathrm{~h}$ ) y de torrencialidad extrema ( $\geq 200 \mathrm{~mm}$ en $24 \mathrm{~h}$ ) a lo largo del año. El estudio del comportamiento de estos fenómenos extremos es de suma importancia en una región como la cuenca del Mediterráneo, donde sus orillas están densamente pobladas; además, es uno de los hot-spots del actual cambio climático (IPCC, 2013). El acotamiento del período de lluvias torrenciales puede ser una aportación valiosa de cara a la planificación y prevención del riesgo en las regiones litorales del Mediterráneo. 


\section{2. ÁREA DE ESTUDIO, DATOS Y METODOLOGÍA}

El área de estudio son las provincias litorales de Cataluña: Girona, Barcelona y Tarragona (Fig. 1). Estas tres provincias se corresponden aproximadamente con la superficie conjunta de las cuencas internas de Cataluña y las tierras del bajo Ebro, y son susceptibles de acumular las mayores precipitaciones del territorio catalán en situaciones de ciclogénesis mediterránea. A partir de distintas fuentes de datos diarios de precipitación (Agencia Estatal de Meteorología, Servicio Meteorológico de Cataluña y Agencia Catalana del Agua, entre otras) se ha elaborado una base de datos de episodios torrenciales ( $\geq 100 \mathrm{~mm}$ en $24 \mathrm{~h}$ ) y torrenciales extremos ( $\geq 200 \mathrm{~mm}$ en $24 \mathrm{~h}$ ) para el período de estudio 1950-2013 (64 años). Se partió inicialmente de 917 registros con $\geq 100 \mathrm{~mm}$ en $24 \mathrm{~h}$ y, tras eliminar episodios dudosos y duplicidad de datos (un mismo observatorio y fecha con dato de precipitación distinto según la fuente), se seleccionaron 862 casos. Se catalogaron todas esas fechas donde en algún observatorio se acumularon $\geq 100 \mathrm{~mm}$, anotándose el lugar y la cuantía máxima. Se obtuvieron 375 fechas en 153 observatorios distintos (Fig. 1). Más del 50\% de los lugares de registro máximo se localizan en la provincia de Girona, sobre todo, en la Sierra Transversal y el Pirineo Oriental. La selección de episodios de $\geq 200 \mathrm{~mm}$ en 24 h fue algo más laboriosa, porque se contrastaron fechas y cantidades con otras fuentes y trabajos (Gázquez y Llasat, 2004). Se obtuvieron 36 fechas con un mínimo de $200 \mathrm{~mm}$ (Tabla 1), con la mayor acumulación de máximos nuevamente en la provincia de Girona (21 casos), seguido de las de Barcelona y Tarragona, con 9 y 6 casos, respectivamente.

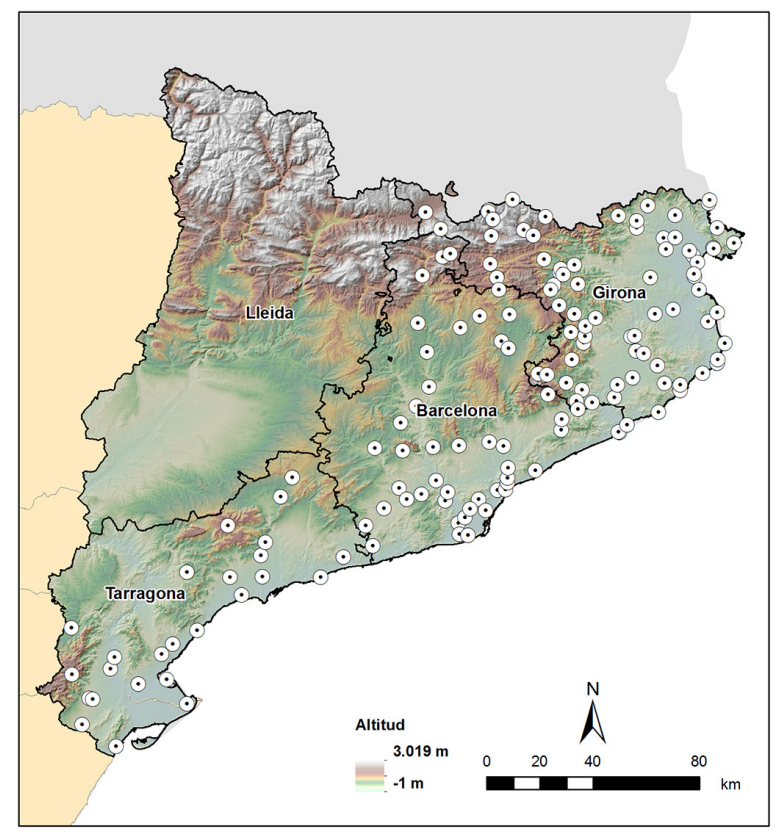

Fig. 1: Localización de los 153 observatorios (círculos) que han registrado al menos una vez la cuantía máxima de un episodio de $\geq 100 \mathrm{~mm}$ en 24 horas a lo largo del periodo de estudio 1950-2013 en las tres provincias costeras de Cataluña. 


\begin{tabular}{|c|c|c|c|}
\hline Fecha & Cantidad & Lugar & Provincia \\
\hline $12 / 10 / 1986$ & 430,0 & Cadaqués & Girona \\
\hline 07/11/1982 & 423,0 & La Molina & Girona \\
\hline $10 / 10 / 1994$ & 397,0 & Cornudella de Montsant & Tarragona \\
\hline $18 / 10 / 1977$ & 385,0 & Vilanova de Sau & Barcelona \\
\hline $07 / 11 / 1983$ & 367,0 & Rubí & Barcelona \\
\hline 20/09/1971 & 308,0 & Esparreguera & Barcelona \\
\hline $20 / 09 / 1972$ & 307,0 & Sant Carles de la Ràpita & Tarragona \\
\hline $23 / 10 / 2000$ & 297,8 & Horta de Sant Joan & Tarragona \\
\hline 03/10/1987 & 291,0 & Castelló d'Empúries & Girona \\
\hline $13 / 10 / 2005$ & 254,4 & Castell d'Aro & Girona \\
\hline $16 / 02 / 1982$ & 251,2 & Amer & Girona \\
\hline $25 / 09 / 1962$ & 250,0 & Martorelles & Barcelona \\
\hline 06/01/1977 & 250,0 & Port de la Selva & Girona \\
\hline $26 / 10 / 1979$ & 250,0 & Calella de Palafrugell & Girona \\
\hline $12 / 11 / 1988$ & 247,3 & Castellfollit de la Roca & Girona \\
\hline $02 / 09 / 1959$ & 246,5 & Cadaqués & Girona \\
\hline 12/11/1999 & 233,5 & Castellfollit de la Roca & Girona \\
\hline $10 / 06 / 2000$ & 233,0 & Rajadell & Barcelona \\
\hline $21 / 12 / 2007$ & 232,9 & Parc Natural dels Ports & Tarragona \\
\hline $06 / 10 / 1959$ & 230,1 & Tossa de Mar & Girona \\
\hline $02 / 10 / 1951$ & 230,0 & Cornellà de Llobregat & Barcelona \\
\hline $21 / 09 / 1959$ & 230,0 & Gualba de Dalt & Barcelona \\
\hline $11 / 10 / 1970$ & 230,0 & Blanes & Girona \\
\hline 04/09/1972 & 227,0 & Sant Feliu de Buixalleu & Girona \\
\hline 26/09/1992 & 226,4 & Amposta & Tarragona \\
\hline $04 / 04 / 1969$ & 226,0 & Rupit & Barcelona \\
\hline $11 / 10 / 1962$ & 223,0 & Sils & Girona \\
\hline $19 / 10 / 1994$ & 220,0 & El Port de Llançà & Girona \\
\hline $17 / 09 / 2010$ & 216,8 & l'Ametlla de Mar & Tarragona \\
\hline $05 / 03 / 2013$ & 214,2 & Viladrau & Girona \\
\hline $11 / 10 / 1990$ & 213,0 & Portbou & Girona \\
\hline $31 / 08 / 1975$ & 208,5 & Santa Agnès de Solius & Girona \\
\hline $13 / 09 / 2006$ & 202,9 & Castelló d'Empúries & Girona \\
\hline $03 / 11 / 2011$ & 202,9 & Òdena & Barcelona \\
\hline $18 / 05 / 1977$ & 200,0 & Roses & Girona \\
\hline $28 / 10 / 1989$ & 200,0 & Port de la Selva & Girona \\
\hline
\end{tabular}

Tabla 1: Catalogación de los 36 episodios de $\geq 200 \mathrm{~mm}$ en 24 h, ordenados de mayor a menor según su valor de precipitación registrado, acontecidos en algún lugar de las tres provincias litorales de Cataluña durante el periodo 1950-2013.

Para el estudio de la variabilidad intraanual de los episodios torrenciales se opta por la construcción de calendarios pluviométricos (Soler y Martín-Vide, 2002). Se cuantifica el número de casos que se acumulan en un determinado espacio temporal del calendario. Este intervalo temporal puede ser de un mes, 15 días, 10 días o menos. En este trabajo se considera el intervalo de 10 días, el cual se corresponde aproximadamente con el período máximo de predicción baroclínica. Se suman las frecuencias de los episodios de $\geq 100 \mathrm{~mm}$ y $\geq 200 \mathrm{~mm}$ por períodos de diez días, que constituyen 36 décadas a lo largo del año, de todo el período de estudio 1950-2013. Estos calendarios pluviométricos permiten delimitar con más precisión que la escala mensual el período de lluvias torrenciales. Asimismo, se contemplan dos subperíodos de 32 años (1950-1981 y 1982-2013) para evaluar posibles desplazamientos temporales en 
el calendario de lluvias torrenciales en las provincias litorales de Cataluña. También se realizan los mismos calendarios para el WeMOi a partir de los valores diarios del índice promediados por períodos de diez días. Finalmente, se solapan los calendarios del WeMOi y de los episodios de precipitación torrencial con el fin de hallar coincidencias en la evolución temporal intraanual de ambos fenómenos.

\section{RESULTADOS Y DISCUSIÓN}

\subsection{Calendario de los episodios de $\geq 100 \mathrm{~mm}$ en $24 \mathrm{~h}$}

Se identificaron 375 fechas con una precipitación $\geq 100 \mathrm{~mm}$ en $24 \mathrm{~h}$ en el período de análisis de 64 años, lo que da un promedio anual en el conjunto de las tres provincias catalanas litorales de 5,9 casos. El número absoluto de episodios torrenciales se mantiene en 15 o más para períodos de diez días desde la primera década de septiembre hasta la segunda década de noviembre, dándose la moda en la segunda década de octubre y una moda secundaria en la segunda década de noviembre (Fig. 2). En las décadas del resto del año el número absoluto de episodios queda por debajo de 15 , a excepción de la primera década de diciembre.

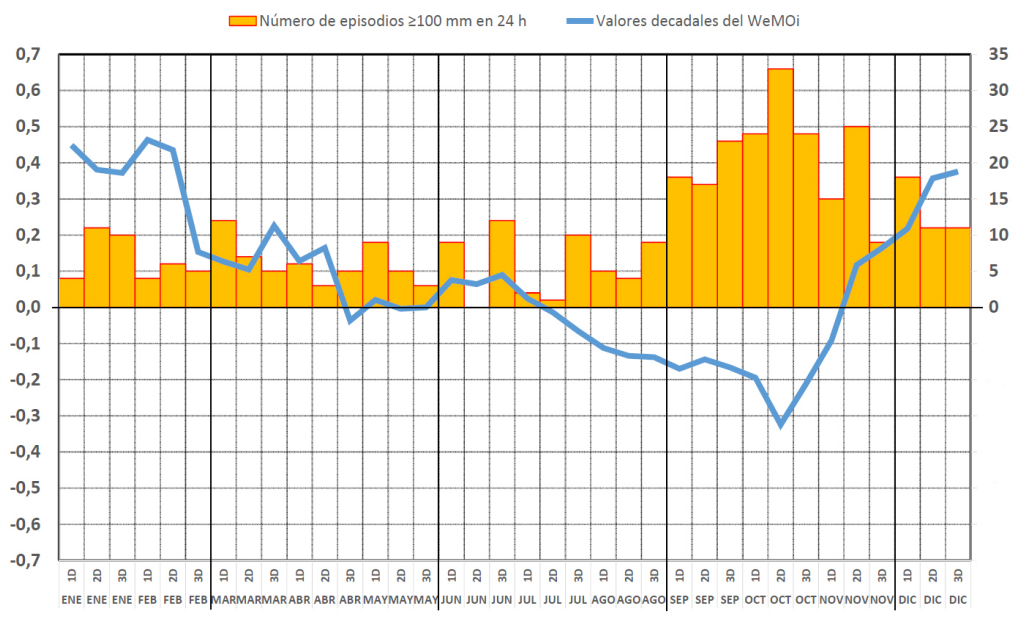

Fig. 2: Calendario por periodos de diez días de los episodios torrenciales ( $\geq 100 \mathrm{~mm}$ en $24 \mathrm{~h})$ acontecidos en las provincias litorales catalanas (eje de ordenadas derecho) y de los valores diarios del WeMOi (eje de ordenadas izquierdo) durante el periodo de estudio 1950-2013.

Los valores decadales del WeMOi son muy positivos al inicio del año y van disminuyendo gradualmente con el transcurso de los meses, con algún repunte a finales de marzo, hasta adentrarse en valores totalmente negativos a finales de julio y principios de agosto. Los valores continúan disminuyendo a finales de verano hasta llegar a un valor mínimo en la segunda década de octubre. Seguidamente, la recuperación es relativamente rápida situándose en valores positivos a mediados de noviembre, y alcanzando valores similares a los de enero en la última década del año. Esta evolución intraanual del WeMOi no muestra desestacionalización debido al proceso de estandarización diaria del índice en que se ha considerado la media y la desviación típica de 
todos los días del año comprendidos en el período de referencia (desde el 1 de enero de 1981 hasta el 31 de diciembre de 2010) (López-Bustins et al., 2014).

Existe una buena correspondencia entre la ocurrencia de los episodios torrenciales y los valores intraanuales del WeMOi (Fig. 2). Es especialmente coincidente la máxima frecuencia absoluta de episodios torrenciales (33) (1 episodio cada 2 años) con el valor mínimo del WeMOi $(-0,32)$ del 11 al 20 de octubre. La ocurrencia de este mayor número de episodios en octubre explica que este mes sea el mes más húmedo en la mayoría de observatorios del litoral catalán y de otras regiones orientales de la Península Ibérica. La mayor frecuencia de fases extremas negativas de la WeMO durante octubre, configuradas por la presencia de altas presiones en Centroeuropa y bajas presiones en el Golfo de Cádiz, favorece la circulación de vientos de levante sobre la Península. Esta circulación mediterránea es el ingrediente principal para favorecer la ocurrencia de episodios torrenciales en el este peninsular (Martín-Vide, 2005). El número de episodios torrenciales presenta un aumento cuadrático en los rangos de valores negativos del WeMOi. El ajuste polinómico de segundo grado es estadísticamente significativo con $\mathrm{r}^{2}=0,3801$ (Fig. 3, izquierda).

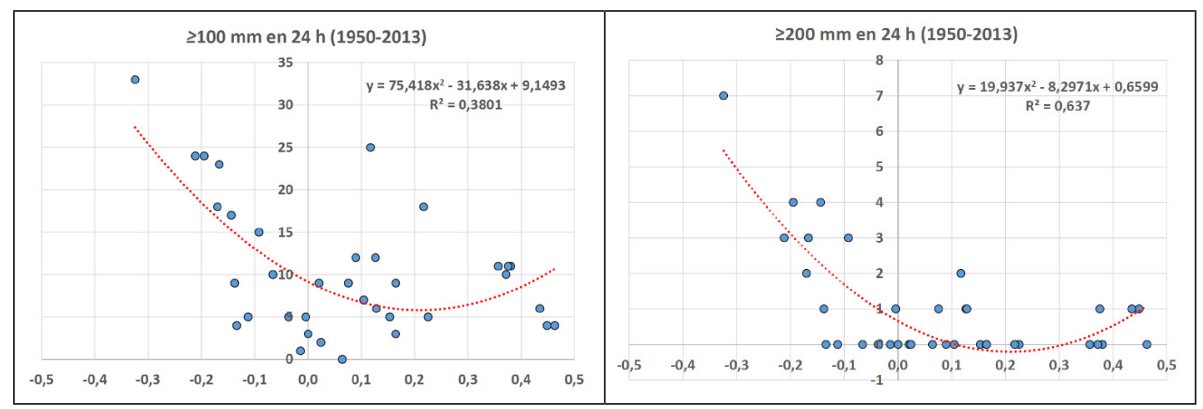

Fig. 3: (Izquierda) Ajuste polinómico de segundo grado del número de episodios torrenciales ( $\geq 100 \mathrm{~mm}$ en $24 \mathrm{~h}$ ) acontecidos en cada década del calendario durante el periodo 1950-2013 y sus correspondientes valores del WeMOi. (Derecha) Ídem que la Fig.3 izquierda, pero para los episodios de torrencialidad extrema ( $\geq 200 \mathrm{~mm}$ en $24 \mathrm{~h})$.

El período intraanual de fase negativa de la WeMO, y propicio a la ocurrencia de lluvias intensas, podría acotarse según los valores del WeMOi por debajo del umbral -0,1, es decir, los meses de agosto, septiembre y octubre (Fig. 2). Es evidente que hay un cierto desajuste entre la frecuencia de episodios torrenciales y los valores del WeMOi en el mes de noviembre. No obstante, si se observan los calendarios por subperíodos (Fig. 4), puede comprobarse que los casos acumulados en las décadas de noviembre, sobre todo del 11 al 20 de noviembre, pertenecen al segundo subperíodo de estudio 1982-2013. En el segundo subperíodo los valores del WeMOi se han tornado especialmente negativos durante la segunda década de noviembre, hecho que podría explicar la acumulación de episodios torrenciales en esos diez días. Se aprecia en los calendarios una disminución generalizada de los valores del WeMOi en todas las décadas del año, que explicaría el mayor número de episodios acontecidos durante el segundo subperíodo 1982-2013 (222) respecto al primero 1950-1981 (153), es decir, un incremento neto de 69 casos entre ambos subperíodos. Un estudio reciente de Lana et al. (2016) muestra una pendiente claramente negativa en 
la serie mensual del WeMOi durante la segunda mitad del siglo XX, que podría estar vinculada a un aumento de la presión atmosférica en superficie a lo largo de las últimas décadas del pasado siglo sobre la Llanura Padana (Maugeri et al., 2004). Este incremento de la torrencialidad también se detectó en un estudio de la Comunidad Valenciana para el período 1959-2000, donde observó un aumento de los episodios asociados a frentes de retroceso (Millán et al., 2005). López-Bustins (2007) analizó los datos del registro más largo de temperatura del mar que se dispone de la costa catalana (desde 1969 en el pueblo gerundense de l'Estartit), y detectó los mayores aumentos entre los subperíodos de 1973-1977 y 1998-2002 durante las décadas de noviembre, especialmente, del 11 al 20 de noviembre (Fig. 5). La combinación en el mes de noviembre de un mar Mediterráneo más cálido frente a la costa catalana, circulación del este en superficie por los valores negativos del WeMOi y presencia de aire polar en altura por las fechas del año, conlleva condiciones muy favorables para la ocurrencia de un mayor número de episodios torrenciales en dicho mes. El calentamiento de las aguas del Mediterráneo también podría haber favorecido un mayor ascenso del aire (menor presión atmosférica en superficie) y contribuir a la disminución de los valores del WeMOi (Perry, 1994; Romeu-Codina y López-Bustins, 2006). El recorrido de los flujos de aire sobre un mar Mediterráneo más cálido también puede conllevar un mayor transporte de energía y vapor de agua e incrementar la torrencialidad de los episodios (Estrela et al., 2008). Se podría intuir un alargamiento del período de lluvias torrenciales hacia el otoño tardío y principios de invierno. Es así que en el segundo subperíodo se registran 34 y 24 episodios en noviembre y diciembre, mientras que en el primer subperíodo estos episodios fueron de 15 y 16, respectivamente. El mes de agosto también se halla en los valores intraanuales del WeMOi 1950-2013 por debajo del umbral - $-0,1$, pero no registra un número destacable de episodios torrenciales. A pesar de las tormentas fuertes que a menudo se producen en este mes, la predominante estabilidad atmosférica en verano reduce la posibilidad de episodios torrenciales de gran cantidad en la región.

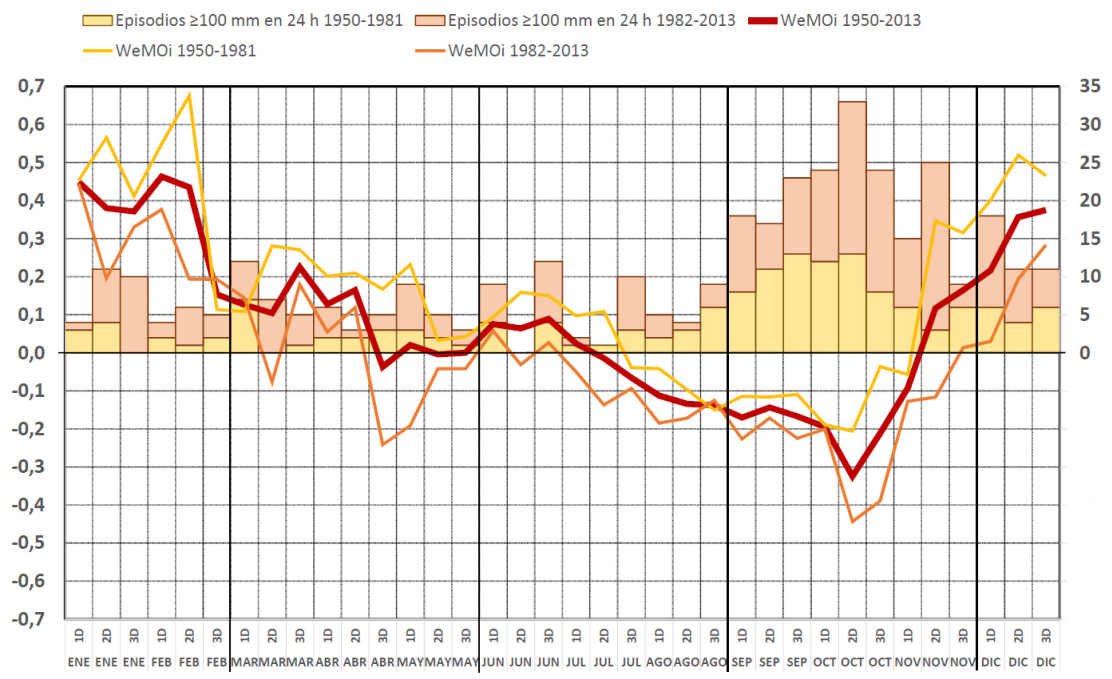

Fig. 4: Ídem que Fig. 2, pero distinguiendo los subperiodos de estudio 1950-1981 y 1982-2013. 


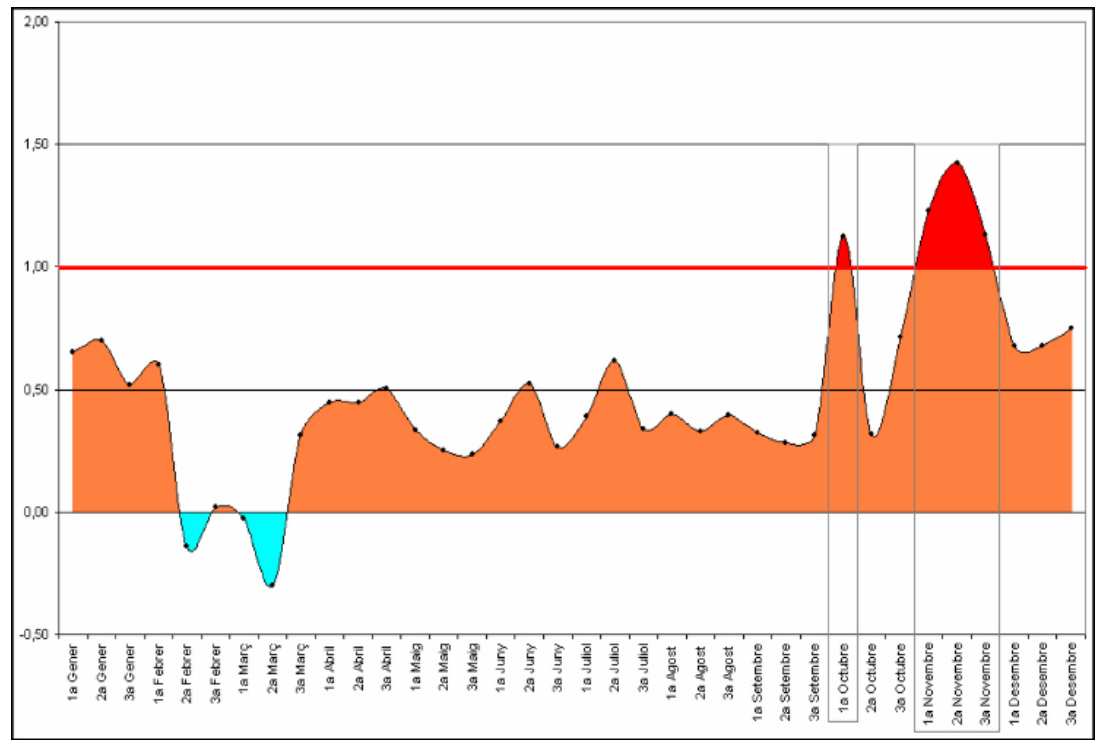

Fig. 5: Representación intraanual de las variaciones de temperatura del agua del mar a 80 metros de profundidad (eje de ordenadas) en l'Estartit (Girona) entre los subperiodos 19731977 y 1998-2002 por periodos de diez días (eje de abscisas). Los valores positivos indican una temperatura más elevada en el subperiodo más reciente. Fuente: López-Bustins (2007).

\subsection{Calendario de los episodios de $\geq 200 \mathrm{~mm}$ en $24 \mathrm{~h}$}

Se identificaron 36 episodios con una precipitación de $\geq 200 \mathrm{~mm}$ en 24 h. El período de retorno de los episodios de $\geq 200 \mathrm{~mm}$ en $24 \mathrm{~h}$ en el conjunto de las tres provincias catalanas litorales es de 1,8 años ( 0,6 casos por año). Esta torrencialidad extrema es menos frecuente que en las provincias vecinas de la Comunidad Valenciana, donde acontecen 1,4 episodios por año (López-Bustins, 2004). Se ha acumulado más de un episodio en las décadas que se suceden desde principios de septiembre hasta mediados de noviembre (Fig. 6). En estas ocho décadas se acumulan 28 episodios (el 78\% del total de casos); los 8 episodios restantes se hallan repartidos entre las otras décadas del año. Las frecuencias de estos episodios de torrencialidad extrema muestran una distribución intraanual similar a la de los episodios de $\geq 100 \mathrm{~mm}$ en $24 \mathrm{~h}$, coincidiendo el máximo en la segunda década de octubre, con 7 episodios (prácticamente el 20\% del total de casos). Este calendario es más nítido que el de los episodios torrenciales (Fig. 2) y permite acotar bien el período de lluvias extremadamente torrenciales desde el 1 de septiembre hasta el 20 de noviembre. La función cuadrática de los episodios de $\geq 200 \mathrm{~mm}$ en $24 \mathrm{~h}$ en relación con los valores del WeMOi decadales muestra una significancia estadística mayor $\left(\mathrm{r}^{2}=0,6370\right)$ (Fig. 3, derecha) que con los episodios de $\geq 100 \mathrm{~mm}$ en $24 \mathrm{~h}$ (Fig. 3, izquierda).

En la distinción por subperíodos de la Fig. 6, se observa que los 5 episodios acontecidos en noviembre pertenecen al segundo subperíodo, donde el WeMOi se ha vuelto mucho más negativo. A pesar de que la máxima frecuencia de episodios ex- 
tremadamente torrenciales tiene lugar del 11 al 20 de octubre en ambos subperíodos, es perceptible un cierto desplazamiento del período de estas lluvias extremas hacia el otoño tardío durante el segundo subperíodo. Durante el primer subperíodo 19501981 los episodios se concentraron desde finales de agosto hasta finales de octubre, mientras que en el segundo subperíodo, 1982-2013, lo hicieron desde mediados de septiembre hasta mediados de noviembre.

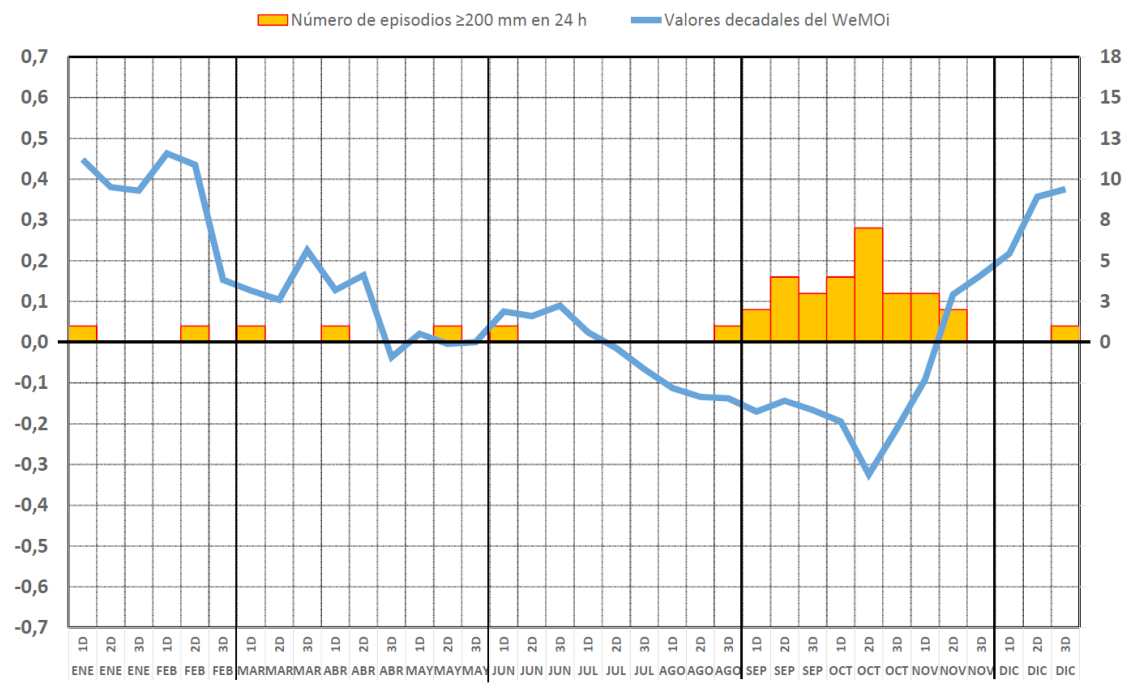

Fig. 6: Ídem que Fig. 2, pero para episodios de torrencialidad extrema ( $\geq 200 \mathrm{~mm}$ en $24 \mathrm{~h})$.

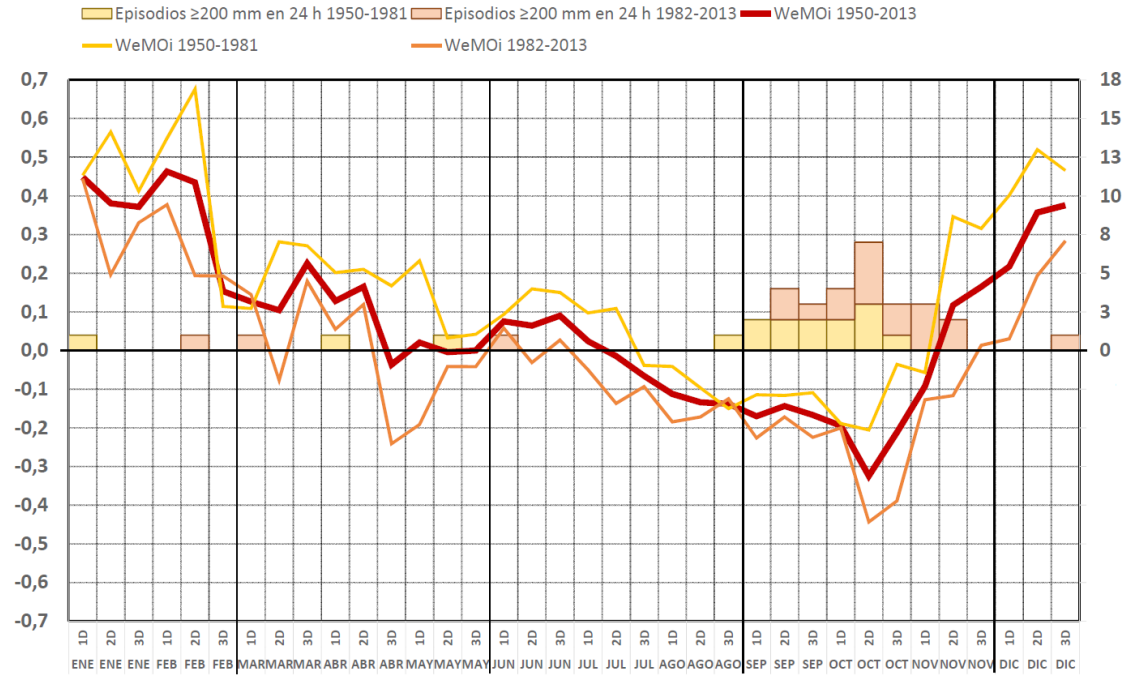

Fig. 7: Ídem que Fig. 4, pero para episodios de torrencialidad extrema ( $\geq 200 \mathrm{~mm}$ en $24 \mathrm{~h})$. 


\section{CONCLUSIONES}

El uso de calendarios de períodos de diez días ha resultado ser una herramienta útil para establecer relaciones entre la ocurrencia de lluvias torrenciales y las fases negativas de la WeMO. En las regiones litoral y prelitoral de Cataluña, los episodios torrenciales ( $\geq 100 \mathrm{~mm}$ en $24 \mathrm{~h}$ ) tienen una frecuencia de ocurrencia de unos 6 casos por año, y en torno a 1 caso cada 2 años en cuanto a los episodios de torrencialidad extrema ( $\geq 200 \mathrm{~mm}$ en $24 \mathrm{~h})$.

Los calendarios pluviométricos para todo el período de estudio 1950-2013 han permitido acotar un período de lluvias torrenciales (con un mínimo de acumulación de 15 casos por década) y torrenciales extremas (con un mínimo de acumulación de 2 casos por década) desde la primera década de septiembre hasta la segunda década de noviembre. La máxima frecuencia de estas precipitaciones torrenciales y torrenciales extremas acontece durante la segunda década de octubre, del 11 al 20 de octubre, con 33 (1 episodio cada 2 años) y 7 casos (1 episodio cada 9 años), respectivamente.

Los valores del WeMOi más negativos para todo el período de estudio 1950-2013 tienen lugar desde la primera década de septiembre (WeMOi -0,17) hasta la última década de octubre (WeMOi -0,21), dándose el mínimo durante la segunda década de octubre (WeMOi -0,32). Hay una evidente coincidencia entre la fase más negativa del año de la WeMO y la ocurrencia de episodios torrenciales en las provincias litorales de Cataluña, dándose el mínimo valor del WeMOi y el número máximo de episodios en la misma década, del 11 al 20 de octubre.

En la comparación de los calendarios de cada subperíodo de estudio, 1950-1981 y 1982-2013, se ha detectado una disminución de los valores del WeMOi a lo largo del año en el subperíodo más reciente. Del mismo modo, los episodios torrenciales y extremadamente torrenciales han aumentado en 69 y 4 casos, respectivamente. Se han reducido especialmente los valores del WeMOi durante la segunda década de noviembre (WeMOi 0,35 para 1950-1981 y WeMOi -0,12 para 1982-2013), en la cual han aumentado muy notablemente el número de episodios torrenciales (3 casos para 1950-1981 y 22 casos para 1982-2013). Este hecho podría estar señalando un posible desplazamiento de este tipo de precipitaciones hacia el otoño tardío.

\section{AGRADECIMIENTOS}

Este trabajo ha sido financiado por el proyecto WEMOTOR (CSO2014-55799C2-1-R, 2015-2017) del Ministerio de Economía y Competitividad, y por el Grup de Climatologia de la Universitat de Barcelona (UB) (2014 SGR 300, Generalitat de Catalunya). Algunos autores son miembros del Institut de Recerca de l'Aigua de la UB.

\section{REFERENCIAS}

De Luis, M., Brunetti, M., Gonzalez-Hidalgo, J.C., Longares, L.A., Martin-Vide, J. (2010). Changes in seasonal precipitation in the Iberian Peninsula during 1946-2005. Global and Planetary Change, 74, 27-33.

Estrela, M.J., Pastor, F., Miró, J., Valiente, J.A. (2008). Precipitaciones torrenciales en la Comunidad Valenciana: La temperatura superficial del agua del mar y áreas de recarga. Primeros resultados. En M.J. Estrela Navarro (Ed.), Riesgos climáticos 
y cambio global en el mediterráneo español ¿hacia un clima de extremos? (pp. 121-140). Valencia: Colección Interciencias.

IPCC (2013). Climate Change 2013: The Physical Science Basis. Contribution of Working Group I to the Fifth Assessment Report of the Intergovernmental Panel on Climate Change. In T.F. Stocker, D. Qin, G.-K. Plattner, M. Tignor, S.K. Allen, J. Boschung, A. Nauels, Y. Xia, V. Bex, P.M. Midgley (Eds.), Cambridge University Press, Cambridge, United Kingdom and New York, NY, USA, 1535 pp.

Kottek, M., Grieser, J., Beck, C., Rudolf, B., Rubel, F. (2006). World Map of the KöppenGeiger climate classification updated. Meteorologische Zeitschrift, 15, 259-263. Retrieved from http://koeppen-geiger.vu-wien.ac.at/pdf/Paper 2006.pdf

Lana, X., Burgueño, A., Martínez, M.D., Serra, C. (2016). Complexity and predictability of the monthly Western Mediterranean Oscillation index. International Journal of Climatology, 36, 2435-2450.

López-Bustins, J.A. (2004). Anàlisi dels episodis extrems de precipitació $\geq 200 \mathrm{~mm} / 24 \mathrm{~h}$ al País Valencià en el període 1951-2000. Ajustament a l'Oscil·lació de la Mediterrània Occidental (WeMO). Trabajo de Máster, Universitat de Barcelona.

López-Bustins, J.A. (2007). The Western Mediterranean Oscillation and Rainfall in the Catalan Countries. Tesis Doctoral, Universitat de Barcelona.

López-Bustins, J.A., Martín-Vide, J., Barriendos, M. (2014). Actualización de los valores del índice de la Oscilación del Mediterráneo Occcidental (WeMOi): 1821-2013. En S. Fernández-Montes, F.S. Rodrigo (Eds.), Cambio Climático y Cambio Global (pp. 283-294). Almería: Publicaciones de la Asociación Española de Climatología (AEC).

Martín-Vide, J. (2002). Ensayo sobre la Oscilación del Mediterráneo Occidental y su influencia en la pluviometría del este de España. En J.A. Guijarro, M. Grimalt, M. Laita, S. Alonso (Eds.), El Agua y el Clima/ L’Aigua i el Clima (pp. 35-42), Palma: Publicaciones de la Asociación Española de Climatología (AEC).

Martín-Vide, J. (2005). Los mapas del tiempo. Mataró: Davinci Continental.

Martín-Vide, J., Olcina-Cantos, J. (2001). Climas y Tiempos en España. Madrid: Alianza Editorial.

Martín-Vide, J., López-Bustins, J.A. (2006). The Western Mediterranean Oscillation and rainfall in the Iberian Peninsula. International Journal of Climatology, 26, 1455-1475.

Maugeri, M., Brunetti, M., Monti, F., Nanni, T. 2004. Sea-level pressure variability in the Po plain (1765-2000) from homogenized daily secular records. International Journal of Climatology, 24, 437-455.

Millán, M., Estrela, M.J., Miró, J.J. (2005). Rainfall Components: Variability and Spatial Distribution in a Mediterranean Area (Valencia Region). Journal of Climate 18, 2682-2705.

Romeu-Codina, E., López-Bustins, J.A. (2006). Aproximación a la posible existencia de la señal de los ciclos solares 18 y 19 en la pluviometría de la Península Ibérica. Geographicalia, 49, 19-36.

Perry, C.A. (1994). Solar-irradiance variations and regional precipitation fluctuations in the western USA. Internationa Journal of Climatology, 14, 969-983. 
Soler, X., Martín-Vide, J. (2002). Los calendarios climáticos. Una propuesta metodológica. En J.A. Guijarro, M. Grimalt, M. Laita, S. Alonso (Eds.), El Agua y el Clima/ L'Aigua i el Clima (pp. 577-586), Palma: Publicaciones de la Asociación Española de Climatología (AEC). 